\title{
Reflets
}

Revue ontaroise d'intervention sociale et communautaire

\section{L'économie en tant que levier de la communauté : Les survivants psychiatriques en Ontario}

\section{Kathryn Church}

Volume 5, numéro 1, printemps 1999

Pratiques et développement économique communautaire

URI : https://id.erudit.org/iderudit/026253ar

DOI : https://doi.org/10.7202/026253ar

Aller au sommaire du numéro

Éditeur(s)

Reflets : Revue ontaroise d'intervention sociale et communautaire

ISSN

1203-4576 (imprimé)

1712-8498 (numérique)

Découvrir la revue

Citer cet article

Church, K. (1999). L'économie en tant que levier de la communauté : Les survivants psychiatriques en Ontario. Reflets, 5(1), 123-144.

https://doi.org/10.7202/026253ar

Tous droits réservés (C) Reflets : Revue ontaroise d'intervention sociale et communautaire, 1999
Ce document est protégé par la loi sur le droit d'auteur. L'utilisation des services d'Érudit (y compris la reproduction) est assujettie à sa politique d'utilisation que vous pouvez consulter en ligne.

https://apropos.erudit.org/fr/usagers/politique-dutilisation/ 


\section{L'économie en tant que levier}

de la communauté

\section{Les survivants psychiatriques en Ontario $^{1}$}

\section{Kathryn Church}

Titulaire d'une bourse de perfectionnement post-doctoral de la Faculté de service social de l'Université de Toronto.

Nous décrivons dans ce texte l'approche axée sur le développement économique adoptée depuis dix ans par les survivants psychiatriques en Ontario ${ }^{2}$. Le terme «survivants psychiatriques» désigne les personnes qu'on appelle généralement les «malades mentaux» ou, simplement, les «fous». Ces personnes sont confrontées à la pauvreté chronique et tentent depuis plusieurs décennies de trouver des méthodes de production de revenus dans leur communauté. Elles ont appris, par essais et erreurs, que l'emploi collectif,par la création d'entreprises communautaires, est la stratégie permettant de répondre le mieux aux besoins des plus vulnérables d'entre elles et de miser sur leurs capacités inexploitées.

Les entreprises communautaires gérées par des survivants psychiatriques ont essentiellement pour but de créer des emplois. Mais elles voient plus loin. Les survivants psychiatriques forment une communauté sociale et politique, bien plus que géographique. Lorsqu'ils s'appliquent à stimuler le développement économique, ils se soucient tout autant de questions telles que l'alphabétisation, le problème des sans-abri, le développement du logement et la pauvreté que de formation, de recrutement de travailleurs et de planification économique.

Indépendamment du produit ou du service qu'ils créent, les survivants psychiatriques entrepreneurs s'emploient également à 
poser des questions concernant les traitements psychiatriques, à rehausser les attentes des survivants psychiatriques face à la vie, à leur offrir de la formation et à leur fournir des occasions de prendre des décisions et d'acquérir des qualités de leader. Autrement dit, plutôt que d'utiliser leur communauté comme un levier de développement économique, ils utilisent l'économie comme un levier de développement communautaire. C'est là l'essence même de leur approche en matière de développement économique.

\section{Une communauté hétérogène}

L'Ontario Council of Alternative Businesses (OCAB) est un organisme parapluie qui regroupe huit entreprises gérées par des survivants psychiatriques et situées dans diverses régions de l'Ontario. En 1994, cet organisme a produit un guide sur le développement des entreprises. Les auteurs du guide soulignent la complexité et l'hétérogénéité de la communauté des survivants psychiatriques. Alors que certains membres croient que les problèmes de santé mentale ont des causes biochimiques, d'autres sont convaincus que la maladie mentale n'existe pas et que le contrôle social est la véritable raison d'être du système de santé mentale. La communauté des survivants psychiatriques est composée d'individus qui ont ainsi des opinions divergentes sur le traitement psychiatrique: certains appuient les pratiques thérapeutiques établies; d'autres souhaitent une modification des pratiques, alors que d'autres encore veulent de véritables solutions de rechange; enfin, certains ne seront satisfaits que lorsque le système existant aura été complètement aboli.

Vu l'hétérogénéité de cette communauté, il est difficile de trouver une appellation unique pour désigner ses membres. L'OCAB encourage donc les gens à s'identifier eux-mêmes en fonction de leur propre point de vue. "L'auto-définition est un moyen de récupérer le pouvoir que nous avons perdu lorsqu'on nous a étiquetés (...). Parmi les termes que nous utilisons figurent «usager (de services)", «usager/survivant», «thriver», «prosumer», 
"...les survivants

psychiatriques ont commencé à se regrouper dans les années 1960, dans la foulée de l'antipsychiatrie ou $d u$ mouvement de libération des survivants psychiatrisés..." «ex-psychiatrisé», «anti-psychiatrie», «fou» et même "postpsychiatrie» (OCAB 1994: 2). Aux fins du présent document, j'utiliserai le terme "survivant psychiatrique». Si cette appellation contribue à «étiqueter» les gens, elle met toutefois l'accent sur leur force. Elle rappelle que les patients ont non seulement survécu à la maladie mentale, mais également aux traitements et aux services psychiatriques - ces deux aspects sont primordiaux pour la communauté des survivants psychiatriques.

Inspirés par des théoriciens comme Erving Goffman et Ronald D. Laing, les survivants psychiatriques ont commencé à se regrouper dans les années 1960, dans la foulée de l'anti-psychiatrie ou du mouvement de libération des survivants psychiatrisés (Burstow et Weitz 1988; Dain 1989). Leurs efforts ont fourni un élan au vaste processus de désinstitutionnalisation qui avait été mis en branle par l'escalade des coûts hospitaliers et facilité par l'arrivée des psychotropes.Au Canada, le retour dans la communauté de centaines d'ex-patients psychiatriques a fait l'objet d'études effectuées par des analystes sociaux et a suscité la production d'innombrables rapports émanant des gouvernements et des universités.

Tous reconnaissent que la communauté n'avait pas les ressources voulues pour aider et appuyer ces personnes qui avaient passé de longues années dans les hôpitaux psychiatriques. Cette réintégration n'avait tout simplement pas été planifiée convenablement et les ressources essentielles n'avaient pas été allouées au secteur communautaire (Trainor et coll.1992). Le corps médical a par ailleurs sous-estimé aussi bien les effets de la vie en établissement que les effets secondaires associés à l'administration prolongée de psychotropes. Pour les survivants psychiatriques, le retour dans la collectivité n'ouvrait pas la porte à l'acceptation ni à l'épanouissement, mais plutôt à une misère écrasante, à l'errance et au rejet social. En un sens, leurs véritables souffrances ont commencé lorsqu'ils ont quitté l'hôpital (OCAB 1995).

Malgré leurs divergences de vue sur le plan idéologique, les survivants psychiatriques sont liés par un destin commun. On les a examinés, on leur a accolé un diagnostic, on les a traités, hospitalisés et soumis à divers traitements; aussi partagent-ils un sentiment profond d'être incompris par les autres membres de la 
«La marginalisation

des survivants

psychiatriques dans

ces secteurs cruciaux

signifie qu'ils sont

généralement, en tant

que groupe, pauvres, sans emploi et mal

logés..." société. Bon nombre de portes se sont fermées devant eux, les empêchant d'exercer pleinement leur rôle de citoyens, et les occasions ratées sur les plans de l'emploi, du logement, de la formation et de la scolarité sont légion. La marginalisation des survivants psychiatriques dans ces secteurs cruciaux signifie qu'ils sont généralement, en tant que groupe, pauvres, sans emploi et mal logés (OCAB 1994, 1995). La participation à la vie économique est essentielle à leur bien-être mais, souvent, les survivants psychiatriques ne disposent pas des moyens les plus élémentaires pour subvenir à leurs propres besoins ou à ceux de leur famille. Alors que des milliers de professionnels de la santé mentale gagnent leur vie dans le secteur des services de santé mentale, les survivants psychiatriques luttent désespérément pour s'intégrer à la vie économique et sociale.

Sous sa forme la plus pure, le regroupement des survivants psychiatriques repose sur la notion d'entraide et fait appel à un réseau variable de groupes auxquels se joignent des gens qui ont en commun leur expérience personnelle des troubles mentaux et des traitements psychiatriques. Le groupe «On Our Own», mis sur pied à Toronto en 1977, est bien connu au Canada. L'un des premiers problèmes abordés par les membres de ce groupe a été la nécessité de gagner de l'argent. À l'époque, comme aujourd'hui, la majorité des membres était sans emploi ou vivait de l'aide sociale ou de prestations d'invalidité. Pour faire face à ce problème, le groupe a organisé un marché aux puces, qui allait devenir «The Mad Market», un magasin sans but lucratif géré par les membres (Burstow et Weitz 1988). Cette entreprise n'existe plus, mais l'idée qui l'a inspirée se perpétue à travers d'autres entreprises gérées par des survivants psychiatriques.

La société ABEL Enterprises, située à Simcoe (Ontario), est la plus ancienne entreprise gérée par des survivants psychiatriques. Au moment de sa création en 1981, elle a pris la forme d'une coopérative de travail dirigée par les membres et se considère aujourd'hui comme un collectif d'entreprise assisté (Church 1987; 1996). Au fil des ans, entre sept et huit entreprises analogues ont emboîté le pas à ABEL. À Toronto, les exemples les plus connus sont $A$-Way Express Courrier Services, créé en 1985, et Fresh Start Cleaning and Maintenance Company, créé en 1990. 
Il n'existe pas de modèle qui permet de décrire précisément le mode de fonctionnement de ce type d'entreprise, et on ne saurait non plus établir aisément une liste des étapes à suivre pour créer une entreprise communautaire. Chacun des groupes, des endroits et des situations a ses particularités et nécessite une approche nouvelle. Il est toutefois possible, à la lumière des expériences antérieures, de dégager des principes généraux et de cerner les composantes habituelles des projets de développement économique communautaire (DÉC).J'exposerai ci-après cinq de ces principes: un processus qui demande de la patience; l'apprentissage actif et l'appui des pairs; des organisations qui favorisent la participation et le droit de regard; la formation d'animateurs et la constitution de réseaux; la fiabilité des partenaires et des mécanismes de financement.

\section{Un processus qui demande de la patience}

Le conseil le plus couramment donné aux personnes désireuses de créer une entreprise communautaire est de concevoir un plan d'entreprise. C'est là, dès le début, que les personnes marginalisées butent sur une profonde contradiction. Bruce Wallace, animateur auprès de la Victoria Street Community Association, explique ainsi cette contradiction: «Pour élaborer un plan d'entreprise, il faut savoir lire et écrire, avoir des connaissances en mathématiques, de l'argent et le sens des affaires. C'est un processus qui risque d'être extrêmement pénible pour des gens qui sont peu scolarisés ou ont une piètre estime d'eux-mêmes et qui sont tenus en marge de la vie économique - et que dire de l'attitude des banques à leur égard. Pour pouvoir élaborer un plan d'entreprise, les membres de la communauté doivent avoir les aptitudes voulues pour occuper un emploi. Or les projets de DÉC visent expressément les communautés qui ont déjà été mises en marge de la vie économique» (Wallace 1996:17).

Les survivants psychiatriques connaissent bien ce dilemme. C'est pourquoi le processus de développement économique 
«La première étape de la création d'une entreprise consiste à découvrir ou à établir la communauté.» débute, pour eux, avant l'étape de la planification, avec la question suivante: "Où est votre communauté»? La première étape de la création d'une entreprise consiste à découvrir ou à établir la communauté. Les survivants psychiatriques franchissent cette étape très simplement en réunissant un certain nombre de personnes qui forment le groupe de base. Par la suite, les membres de ce groupe se rencontrent, échangent, se découvrent mutuellement, apprennent à connaître les compétences et les capacités de chacun et prennent des décisions sur la façon dont ils entendent procéder.

Cela peut sembler relativement simple et, d'une certain façon, c'est simple. Mais, encore une fois, en raison des conditions de vie difficiles et de la vulnérabilité personnelle des participants, le processus peut être exigeant et laborieux. Pour bien fonctionner, le développement d'entreprises par des survivants psychiatriques doit passer par une transformation des attentes: il faut faire en sorte que des gens qui attendent peu, voire rien, de la vie se mettent à en attendre quelque chose. Développer une entreprise avec les membres de cette communauté, c'est entreprendre le travail délicat qui les amènera à troquer leur identité de patients contre celle d'employés. Ce processus exige une grande patience.

Dans la majorité de ces entreprises, les employés peuvent, sur demande, obtenir des congés sans risquer de perdre leur emploi ou d'être jugés irresponsables; l'absentéisme planifié est accepté. Les survivants psychiatriques trouvent que cette approche les aide énormément (Church et Creal 1995a; 1995b). En ayant la possibilité de modifier leur horaire de travail, de prendre des périodes de répit au besoin ou de consacrer leurs «meilleures heures» au travail, les survivants psychiatriques peuvent préserver leur équilibre sur les plans physique et psychologique. Cette souplesse est le témoignage de patience le plus élémentaire de ces entreprises envers leurs employés.

Ces entreprises reconnaissent en outre le fait qu'il faut du temps aux survivants psychiatriques pour apprendre à gérer une entreprise communautaire. Bon nombre d'entre eux ne sont familiers qu'avec la prise de décisions autoritaire. Lorsqu'ils commencent à prendre des décisions concernant leur entreprise, ils adoptent souvent le même style autoritaire auquel on les a 
habitués au cours de leur passage dans le système de santé mentale. Il se peut qu'ils prennent, au début, des décisions qui ne leur sont pas avantageuses. Ils établissent parfois des règles de comportement ou de rendement extrêmement rigoureuses pour s'apercevoir après coup qu'ils enfreignent eux-mêmes les règles et doivent en subir les conséquences. La modération vient avec l'expérience, mais il faut parfois des années avant qu'elle soit acquise.

Les projets de DÉC gérés par des survivants psychiatriques sont caractérisés par la lenteur de leur croissance. Toutefois, les projets mis en oeuvre par cette communauté ne sont pas les seuls à présenter cette caractéristique. Selon le théoricien australien Paul Wildman, «tout projet de DÉC est un projet à long terme. Les organisations participantes doivent s'y engager pour longtemps». Il suggère aux participants d'établir un équilibre entre les initiatives à court terme (un à deux mois), les résultats à moyen terme (un an) et l'échéance à long terme (cinq à dix ans) (Wildman 1993). Les chercheurs américains O'Reagan et Conway sont du même avis que Wildman; selon eux, les réussites dans ce domaine ne se sont pas bâties en un jour. Il faut des années avant qu'un projet mûrisse et devienne une entreprise viable, capable de fournir un réel appui économique aux membres des communautés défavorisées. «Il n'existe pas de méthode miracle permettant de mettre sur pied des projets durables pour vaincre la pauvreté» (O’Reagan et Conway 1993: 31).

"...c'est en faisant

face aux divers problèmes qui se présentent et en tentant de les résoudre que les survivants psychiatriques de retour an travail acquièrent un sentiment d'appartenance à la communauté.»
Les faux pas sont nombreux au cours de la mise sur pied d'une entreprise gérée par des survivants psychiatriques et une fois que l'entreprise est en place et fonctionne, il faut encore traverser régulièrement des périodes de crise. Toutefois, c'est en faisant face aux divers problèmes qui se présentent et en tentant de les résoudre que les survivants psychiatriques de retour au travail acquièrent un sentiment d'appartenance à la communauté. À ce sujet, Jacques Tremblay dira tout simplement qu'il s'agit de «passer au travers». Cet animateur, qui a appuyé la mise sur pied de nombreux projets en Ontario, sait que les survivants psychiatriques traversent un processus de développement non seulement au début mais à de nombreuses étapes de la vie de l'entreprise. Une fois qu'une communauté a été formée, il faut éviter que le 
stress engendré par la direction de l'entreprise ne la fasse éclater. Il faut, sciemment, tenter de renforcer les liens entre les employés en organisant des fêtes, en favorisant les rencontres impromptues et les conversations informelles entre les membres. Les survivants psychiatriques entrepreneurs considèrent l'épanouissement du groupe comme une composante légitime et permanente de l'épanouissement de l'entreprise.

\section{L'apprentissage actif et la formation par les pairs}

Les survivants psychiatriques et les personnes vivant dans la pauvreté ont des aptitudes que bon nombre de gens dits normaux n'ont pas toujours. La plupart des gens «normaux» n'arriveraient pas à surmonter les difficultés auxquelles sont confrontés de nombreux survivants psychiatriques, au dire de Diana Capponi, coordonnatrice à OCAB.

Il y a plusieurs années, un groupe appelé le Toronto Community Economic Development Network a procédé à une étude qualitative d'une durée de deux ans qui visait à déterminer les répercussions des projets de DÉC sur la vie des gens qui doivent surmonter de nombreux obstacles à l'emploi. Les survivants psychiatriques et les entreprises gérées par ces derniers ont été intégrés à cette étude. L'une des conclusions importantes de cette recherche a été que les répondants les plus satisfaits de travailler dans ces entreprises étaient ceux qui les considéraient comme des lieux d'apprentissage (Church et Creal 1995b). Ils disaient y acquérir des habiletés et des compétences nouvelles, y voyaient une occasion d'élargir leurs intérêts et d'accroître leur capacité de s'adapter à des situations inconnues. Pour certains, le simple apprentissage du travail constituait un défi personnel qui monopolisait presque toute leur énergie. D'autres avaient acquis une telle maitrise de leur travail qu'ils pouvaient également devenir des chefs et des décideurs au sein de l'entreprise. Ce processus supposait l'acquisition de compétences supplémentaires; ces personnes devaient également avoir la confiance et le courage voulus pour parler haut et fort. 
"N'attendez pas

d'avoir les

connaissances voulues.

Agissez maintenant.

Puis réfléchissez et

apprenez. Puis

agissez de nouveau.

Ce sont vos actions

qui sont fécondes».
Chose passablement étonnante, il est rare que la littérature relative au DÉC considère l'apprentissage comme une composante fondamentale de cette sphère d'activité. Un article de Paul Wildman fait exception. L'auteur y décrit un plan conceptuel qu'il appelle le «modèle de DÉC à trois composantes» (Wildman 1996). Ces trois composantes sont l'économie régionale, le développement communautaire et l'apprentissage par l'action. La formule proposée parWildman pour favoriser l'apprentissage dans le cadre du DÉC est remarquablement simple: «N'attendez pas d'avoir les connaissances voulues. Agissez maintenant. Puis réfléchissez et apprenez. Puis agissez de nouveau. Ce sont vos actions qui sont fécondes». Cet énoncé constitue une ardente invitation, dont nous avions d'ailleurs grand besoin, à ne plus dépendre des conseils des experts qui ne sont pas toujours disponibles, mais plutôt à apprendre par l'action. Wildman ajoute: «Il faut, pour cela, avoir la force d'admettre ses erreurs et d'apprendre à travers ses erreurs». Autrement dit, de progresser à partir de ses échecs («fail forward») (Wildman 1993: 7).

Progresser à partir de ses échecs.Voilà un concept avec lequel on est familier dans les entreprises gérées par des survivants psychiatriques. Dans l'étude effectuée par le Community Economic Development Network, on relève des exemples, au hasard: c'est Marc Lafontaine qui exploite presque littéralement «à même son portefeuille» la nouvelle compagnie Fresh Start Cleaning; c'est Paul Quinn, du Gerstein Crisis Centre, qui dit à Marc: «Envoie-nous simplement quelqu'un, et nous allons essayer d'arranger tout ça»; c'est Robert O'Connell qui affirme que l'entreprise From the Root Landscaping s'est développée "comme un plat de spaghettis tombant sur le sol» (Church et Creal 1995a). Les personnes qui ont contribué à faire progresser ces entreprises, et d'autres comme elles, n'ont pas hésité, malgré leur expertise limitée, à entreprendre le travail qui devait être fait. Elles ont pris le risque de commettre des erreurs, ont appris à travers leurs erreurs et sont allées de l'avant, conformément au slogan de la société Nike : «Just Do It» (Fais-le!).

Par ailleurs, comme beaucoup d'autres travailleurs dans le contexte de la nouvelle économie, les survivants psychiatriques 
ont des besoins pressants en matière de formation/ perfectionnement et d'acquisition des compétences. Les fonds dont disposent actuellement les divers gouvernements dans ce domaine sont généralement octroyés à d'autres groupes. Les survivants psychiatriques sont souvent éliminés de façon officieuse, parce que leur allure singulière et les comportements qu'ils ont acquis en institution donnent l'impression qu'ils ont peu de chance de réussir. Ceux qui sont admis aux programmes de formation s'avèrent souvent incapables d'apprendre dans le cadre structuré d'une classe. L'OCAB a fait pression pour que des fonds soient consacrés expressément à la création de programmes destinés aux survivants psychiatriques. Ces efforts n'ont pas porté fruit jusqu'ici; les entreprises ont pris en charge le problème en mettant sur pied de la formation par les pairs.

Les survivants psychiatriques qui prennent en charge le développement économique ont appris que la meilleure façon de déterminer si une personne peut faire un travail, c'est de lui confier ce travail en lui donnant également toute l'aide nécessaire pour l'accomplir. Dans les entreprises gérées par des survivants psychiatriques, il n'y a pas de professionnels payés pour procéder à une évaluation des compétences ou dispenser de l'enseignement aux personnes qui n'ont pas les compétences voulues. L'enseignement est plutôt un processus continu, ancré dans la pratique. Des personnes qui ont été des usagers du système de santé mentale et qui ont connu la rue expliquent dans leurs propres mots à d'autres

"Les employés s'autoresponsabilisent et réagissent aux contraintes qu'ils s'imposent mutuellement.» personnes, dont le destin a été identique, comment accomplir un travail. Les employés s'auto-responsabilisent et réagissent aux contraintes qu'ils s'imposent mutuellement. Si, par exemple, seulement la moitié d'une équipe de nettoyage se présente chez un des clients de Fresh Start, elle devra accomplir seule tout le travail. Il y a fort à parier que les personnes présentes feront savoir aux absents ce que cela a représenté d'accomplir seuls tout le travail. Par conséquent, les commentaires des collègues de travail et le risque de fermeture de l'entreprise deviennent des éléments de motivation.

Le concept de «déprofessionnalisation» inhérent à cette approche, qui n'est pas sans rappeler l'anti-psychiatrie, est préoccupant pour les spécialistes de la réadaptation professionnelle. 
Non seulement cette approche récuse-t-elle la façon dont ces spécialistes gagnent leur vie, mais elle remet également en question l'importance qu'ils accordent à la normalisation et à l'intégration. L'intégration ne constitue pas l'objectif premier des entreprises gérées par des survivants psychiatriques; les animateurs ne se soucient pas de porter au maximum la durée des interactions entre leurs employés et les personnes non handicapées. La raison d'être de ces entreprises, c'est d'offrir aux survivants psychiatriques un lieu et un moment où ils peuvent se rencontrer et établir des relations en tant que pairs et collègues de travail. Leur existence permet aux survivants psychiatriques de parler leur propre langage, à leurs propres fins, sans l'intervention des professionnels, sans volonté d'intégration. Les liens qui se trouvent renforcés, ce ne sont pas les liens avec l'ensemble de la société ni avec les dispensateurs de services professionnels, mais plutôt les liens avec le mouvement d'entraide en santé mentale et ceux qui en font partie.

Cette attitude ne contredit nullement le fait que les projets de DÉC gérés par des survivants psychiatriques constituent un vibrant réquisitoire contre la ghettoïsation. C'est l'idée qui me vient à l'esprit chaque fois que je rencontre des messagers d'A-Way Express au travail. Si je ressens un tel plaisir à la vue de leur sac de livraison, c'est parce que je sais que leur présence dans le métro est un exemple extraordinaire de véritable intégration. Dans la lutte contre la stigmatisation, chaque messager est le meilleur agent de relations publiques qui soit.

\section{Des organisations qui favorisent la participation et le droit de regard}

Dès sa création, A-Way Express a connu le succès, car ce sont les gens qui avaient besoin d'un emploi qui ont déterminé euxmêmes comment l'entreprise serait mise sur pied, comment elle fonctionnerait et qui y participerait.A-Way a progressé à tel point qu'elle est maintenant gérée et exploitée à $100 \%$ par des usagers 
«Deux éléments caractérisent une entreprise communautaire: le fait d'être une entreprise sans but lucratif ou appartenant à des travailleurs et la gestion participative.» ou des survivants psychiatriques. C'est un point crucial. C'est ce qui les distingue des autres projets semblables actuellement mis sur pied par des établissements, selon Laurie Hall, directrice administrative, A-Way Express Couriers.

Deux éléments caractérisent une entreprise communautaire : le fait d'être une entreprise sans but lucratif ou appartenant à des travailleurs et la gestion participative. Idéalement, ces caractéristiques doivent évidemment être présentes. En faire une réalité n'est toutefois pas chose facile. Bruce Wallace rappelle que le DÉC est un processus à ce point exigeant, dans les faits, que les personnes mêmes que l'on entend intégrer au processus s'en trouvent exclues. Pour entreprendre le vaste exercice de planification qui s'impose, il faut faire appel à des participants qui ont de l'énergie et de l'enthousiasme à revendre. Le temps et la stabilité sont des éléments déterminants au cours d'un processus de développement fastidieux et extrêmement bureaucratique, marqué par les déconvenues et les impondérables. Comme l'a souligné Wallace, avec une ironie amère, ce n'est guère le type d'entreprise qui convient à des gens vivant dans les quartiers pauvres de la ville, «d'un chèque d'aide sociale à l'autre», et aux prises avec des problèmes de santé mentale et de toxicomanie. En effet, conclut-il, ce type de projet convient bien davantage aux "consultants, comptables, avocats et travailleurs communautaires» (Wallace 1996:18). Comment expliquer, alors, que des entreprises gérées par des survivants psychiatriques aient pu voir le jour en Ontario?

Il a fallu au moins une décennie de travail, en grande partie non reconnu, de la part de dirigeants du mouvement des survivants psychiatriques, de professionnels de la santé mentale alliés et d'organisateurs communautaires pour créer un milieu favorable aux projets d'entreprises gérées par des survivants psychiatriques. Le travail novateur accompli dans le domaine de la participation des usagers par l'Association canadienne pour la santé mentale, en collaboration avec les dirigeants du mouvement, s'est soldé par l'élaboration d'un cadre conceptuel que l'on retrouve presque intégralement dans le plan directeur du gouvernement ontarien pour un système communautaire de santé mentale (Trainor et Church 1984; Hutchison et coll. 1986; Pape 1988; Ontario 1988). 
Pour la première fois, les survivants psychiatriques ont été directement associés à la mise en oeuvre de ce plan et ont notamment participé à une consultation provinciale portant sur la législation relative aux services communautaires de santé mentale.

Cette consultation a eu principalement pour effet de sensibiliser, au moins temporairement, la «bureaucratie de la santé mentale» aux problèmes des survivants psychiatriques. À l'issue de cette consultation, le gouvernement ontarien a octroyé 3,1 millions de dollars en financement anti-récession pour un projet spécial à l'intention des survivants psychiatriques. Ces fonds ont permis de mettre sur pied la Consumer/Survivor Development Initiative (CSDI) (Church 1993). S'inspirant de critères qui privilégiaient les activités non traditionnelles, la gestion par les survivants psychiatriques et un mécanisme démocratique de régie par les membres, la CSDI a financé 42 projets soumis par des groupes de survivants psychiatriques au cours de sa première année de fonctionnement; sept d'entre eux étaient des projets de développement économique (CSDI 1992).

Le mécanisme formel auquel on a recours dans le cadre de ces initiatives et d'autres initiatives économiques pour favoriser la prise en charge et la gestion participative, c'est la participation au conseil d'administration. La structure actuelle d'A-Way Express, par exemple, comprend un conseil d'administration, une équipe de gestion et les membres du personnel. La moitié des dix membres du conseil d'administration sont des employés. L'autre moitié est composée de représentants de l'extérieur qui ont des compétences dans le monde des affaires ou de la gestion des organismes sans but lucratif. Ces personnes sont parfois, mais pas toujours, des survivants psychiatriques. L'équipe de gestion comprend les chefs de bureau et les responsables du marketing, le directeur administratif, le répartiteur en chef, le responsable des finances, le préposé à la prise des commandes téléphoniques et deux représentants des messagers. Depuis sa création en 1985, A-Way réunit une fois par mois tous les membres de son personnel pour

"C'est le personnel qui dispose du pouvoir de décision finale...» leur donner l'occasion d'aborder les questions qui les préoccupent et de faire des recommandations. C'est le personnel qui dispose du pouvoir de décision finale, étant donné qu'il est représenté à 
tous les paliers de l'entreprise et qu'il est associé à l'examen officiel des objectifs immédiats et à long terme lors des assemblées générales annuelles (OCAB 1994; Church 1996).

Officiellement constituée en coopérative de travail sans but lucratif en 1983, ABEL Enterprises était familière avec la gestion participative bien avant que le mot «habilitation» ou empowerment ne devienne à la mode dans le secteur des services sociaux et avant que le mouvement des survivants psychiatriques n'ait des assises solides en Ontario. La leçon principale qu'il convient de tirer de cet effort, c'est qu'en l'absence de structures et de processus distincts, la notion même d'entreprise gérée par des survivants psychiatriques n'est guère plus que de la poudre aux yeux.

Pour ABEL, c'est le comité de planification et de développement, dont tous les employés sont membres, qui constitue la structure clé. Ce comité a son propre président, et le conseil d'administration le laisse entièrement libre d'agir conformément à son mandat. Il est l'élément moteur du conseil d'administration, composé de survivants psychiatriques $(50 \%)$ et de membres de la collectivité $(50 \%)$. Il incombe aux membres du personnel d'organiser le comité et de veiller à la communication de ses recommandations au conseil d'administration. Le comité de planification et de développement a décidé récemment de modifier les pratiques commerciales pour passer d'un modèle coopératif à un modèle d'entreprise. Actuellement, $A B E L$ Enterprises se définit comme un collectif d'entreprises assistées qui aide les membres à acquérir des compétences et à développer des produits. Les membres du personnel invitent les gens à utiliser leurs installations pour lancer leurs propres projets économiques.

\section{La formation d'animateurs et la constitution de réseaux}

"''ai appris il y a longtemps que ce qui fait le plus de bien aux personnes misérables, désorientées et exploitées, c'est de voir que l'une d'entre elles peut se tenir debout et parler haut et fort sans 
"Quand ils comprennent que c'est possible, les gens se sentent revalorisés.» être aussitôt rappelée à l'ordre. Quand ils comprennent que c'est possible, les gens se sentent revalorisés. Après quelque temps, ils s'aperçoivent qu'ils peuvent en faire autant. J'ai voulu favoriser ce type de processus qui s'amorce lorsque les gens comprennent qu'ils pourront susciter des changements à l'intérieur du système et à l'intérieur d'eux-mêmes s'ils osent prendre la parole pour défendre leurs intérêts et ceux des autres» (Pat Capponi, citée dans Church 1991:6).

Il y a quelques années, sous l'égide du Psychiatric Survivor Leadership Facilitation Program, Pat Capponi a conçu un certain nombre d'exercices pratiques visant à guider ses camarades survivants psychiatriques dans le monde mystérieux des dispensateurs de services. En faisant appel au jeu de rôle et à l'analyse de situations, elle les a invités à analyser en petits groupes les questions qui les touchaient de près. Son approche avait essentiellement pour but d'«aider les membres à découvrir leurs propres capacités et possibilités inexploitées et à les renforcer par l'établissement de liens affectifs et de communications avec le groupe» (Church 1991 : 7).

Pat accordait une grande importance à l'établissement de liens de confiance entre les membres du groupe, condition essentielle à la révélation de soi. Elle invitait les membres du groupe à écouter avec une grande ouverture d'esprit les récits des autres participants concernant le système de santé mentale et, indépendamment de la teneur des propos, à valoriser l'expérience de chacun et à s'en inspirer. L'une de ses principales découvertes a été de reconnaitre que les participants avaient besoin de groupes d'entraide parallèles mais distincts. Il se passait trop de choses à l'intérieur des groupes pour que les séances de formation suffisent à les intégrer entièrement. Il était important de mettre les nouveaux animateurs en relation avec les animateurs et les groupes existants (Church 1991).

Dans la même veine, les entreprises gérées par les survivants psychiatriques ne peuvent pas progresser sans un solide esprit d'entraide réciproque. Il importe qu'elles nouent des liens avec d'autres entreprises semblables, aussi bien à l'échelle municipale et régionale que provinciale. Depuis 1992, l'OCAB facilite la constitution de tels réseaux. Il s'attache principalement à aider 
avec la résolution des problèmes pratiques entourant le développement de l'entreprise. Il s'emploie en outre à créer et faire connaitre des ressources qui lui permettent de remplir son rôle dans les domaines de l'éducation et de la défense des droits. En tant que porte-parole des entreprises gérées par des survivants psychiatriques en Ontario, l'OCAB a établi divers types de partenariats et a été associé à l'élaboration des politiques. Les entreprises existantes ont délégué des représentants au sein des comités gouvernementaux; elles y ont gagné en visibilité, et ces représentants ont pu acquérir une expérience précieuse.

Ainsi, l'OCAB a pu poursuivre le travail amorcé par le Leadership Facilitation Program. Il est devenu une tribune où les survivants psychiatriques tentent de s'élever en faux contre les appellations, définitions et interprétations qui ont été générées à leur sujet par le système traditionnel de services psychiatriques. Dans sa documentation, l'OCAB affirme son appui aux options proposées en remplacement des modèles de traitement médicaux/ cliniques - des options qui permettraient aux survivants psychiatriques de ne plus se considérer uniquement comme des «malades». L'OCAB considère le DÉC comme une forme d'intervention en santé mentale dont la structure permet de tenir compte des comportements et des limites des survivants psychiatriques, de leur offrir de l'éducation et de la formation et d'aider les employés à nouer des liens aussi bien les uns avec les autres qu'avec leur communauté. Dans la mesure où ce Conseil et ses membres réussissent dans cette entreprise, ils favorisent la formation d'animateurs issus du milieu.

Les entreprises gérées par les survivants psychiatriques

"...les entreprises communautaires permettaient à leurs employés d'avoir accès non seulement à un lieu du travail, mais également à un lieu de prise de décisions.» contribuent bel et bien à découvrir et à former des animateurs. L'une des conclusions importantes de la recherche effectuée par le Community Economic Development Network était que les entreprises communautaires permettaient à leurs employés d'avoir accès non seulement à un lieu du travail, mais également à un lieu de prise de décisions. Dans le meilleur des cas, ces entreprises constituaient des forums où des personnes non familières avec la prise de décisions apprenaient à jouer ce rôle. Les répondants ont indiqué que c'était important pour eux de sentir que l'on 
dépendait d'eux plutôt que d'être eux-mêmes dans une situation de dépendance. Pour certains, cette expérience a débouché sur une participation plus importante, car les entreprises s'échangeaient les membres de leurs conseils d'administration; les organismes de santé mentale puisaient dans ce réservoir les usagers de services expérimentés appelés à faire partie de leur propre structure organisationnelle. Les employés qui avaient participé à une entreprise communautaire finissaient souvent par participer plus activement aux activités des mouvements de défense des intérêts des survivants psychiatriques. La participation à un projet de DÉC présentait notamment l'avantage, pour les survivants psychiatriques eux-mêmes, de pouvoir organiser leur travail de manière à se réserver du temps pour des engagements sociaux et politiques de plus grande envergure (Church et Creal 1995b).

\section{La fiabilité des partenaires et des mécanismes de financement}

"Tant que le système fonctionnera comme il fonctionne actuellement, l'appui financier devra être permanent»...»
«Bon nombre de gens ont l'impression qu'il suffit d'octroyer un peu de capitaux de démarrage à des projets économiques pour que, quelques années plus tard, ces entreprises s'auto-financent et n'aient plus besoin d'appui extérieur. Ils se trompent. Tant que le système fonctionnera comme il fonctionne actuellement, l'appui financier devra être permanent» (Mary Taylor, directrice administrative, ABEL Enterprises).

Les entreprises gérées par des survivants psychiatriques qui ont été les plus stables et ont connu le plus de succès en Ontario sont celles qui ont noué des liens fonctionnels avec des personnes de leur région qui avaient de l'expertise sur les plans technique et organisationnel. De plus en plus, ces personnes-ressources sont des survivants psychiatriques, mais on fait aussi appel à des membres de la collectivité en général. Ces personnes offrent leur expertise en agissant comme consultants ou comme membres du conseil d'administration et, à l'occasion, comme membres du personnel. 
Pour ABEL Enterprises, par exemple, il a été fondamental d'embaucher les bons employés. Plutôt que de faire appel à des professionnels de la santé mentale, l'entreprise a embauché un designer-ébéniste, un travailleur de la fibre de verre et un architecte paysagiste. Le personnel d'ABEL Enterprises, qui compte davantage d'artistes que de cliniciens, a des compétences très diversifiées dans des domaines comme la charpenterie, le travail du bois, la soudure, l'aménagement paysager, l'art, la tenue de livres et la planification d'entreprise.

L'un des problèmes auxquels sont régulièrement confrontés les survivants psychiatriques qui deviennent productifs est le fait qu'ils sont eux-mêmes un «lieu de production» pour les professionnels de la santé mentale. Désireux d'échapper à cette relation, ils se montrent très prudents lorsqu'ils établissent des partenariats avec les organismes de santé mentale. Selon l'OCAB, les bons partenaires sont les groupes ou les organismes communautaires qui offrent des locaux aux survivants psychiatriques, leur permettent d'avoir accès au matériel de bureau et au café. Ils aident à recruter les gens au cours du démarrage du projet. À mesure que l'entreprise évolue, ces partenaires offrent leur expertise dans des domaines tels que la création d'un conseil d'administration ou la gestion du personnel. Une bonne préparation permet d'éviter les luttes de pouvoir qui surviennent généralement au cours de ce processus. Les partenaires potentiels devraient prendre le temps de se connaitre mutuellement, de préciser les rôles de chacun et de déterminer à quel moment l'entreprise gérée par les survivants psychiatriques deviendra

"...il est essentiel que l'organisme d'appui sache comment se retirer de l'entreprise en temps et lieux.» autonome. L'entente devrait être exposée en détail dans un contrat écrit. En fin de compte, il est essentiel que l'organisme d'appui sache comment se retirer de l'entreprise en temps et lieux.

Si cette capacité de «lâcher prise» est la qualité que les survivants psychiatriques apprécient le plus chez leurs partenaires, la persévérance vient au second rang. Cette qualité est tout particulièrement recherchée chez les partenaires financiers. Les auteurs d'une récente étude sur le développement économique, menée auprès de groupes marginalisés, ont souligné l'importance de la constance et de la souplesse du mécanisme de financement et la 
nécessité que les donateurs maintiennent leur appui même dans les moments difficiles (O'Reagan et Conway 1993). Au cours des dix dernières années, le ministère de la Santé de l'Ontario a joué ce rôle auprès des entreprises gérées par des survivants psychiatriques. Il a octroyé des subventions pour couvrir les coûts administratifs, alors que les revenus générés par les employés servaient à payer les salaires. Ce mécanisme novateur, qui repose à la fois sur des fonds publics et privés, reconnait l'importance de ces entreprises qui endossent les responsabilités à long terme de la société à l'égard d'un groupe vulnérable.

Les entreprises gérées par les survivants psychiatriques subissent des pressions croissantes de la part des gouvernements désireux de les voir s'autofinancer. Malgré ces pressions, elles demeurent fidèles à leur engagement d'embaucher les membres les plus défavorisés de leur communauté. Elles estiment en effet que l'argent investi par le ministère de la Santé dans ce processus permet à la population de s'acquitter de ses responsabilités envers les personnes vulnérables, d'une façon productive, qui représente une nette amélioration par rapport aux programmes de réadaptation professionnelle. Les entreprises peuvent tenter de devenir plus concurrentielles sur le marché, mais il reste que la majorité de leurs employés sont des personnes dont la vie a été gravement perturbée. Par conséquent, si le rapport entre les subventions et les revenus générés peut changer au fil du temps, les entreprises gérées par les survivants psychiatriques auront sans doute toujours besoin d'une certaine forme d'aide financière pour survivre.

\section{Conclusion}

De façon générale, le développement économique par les survivants psychiatriques est un processus volontaire et continu axé sur la communauté locale. Il passe par le respect mutuel, la réflexion critique, l'empathie et la participation des groupes. Ce processus permet à des personnes qui n'ont pas eu leur juste part des ressources importantes d'avoir plus largement accès à ces ressources et plus d'emprise sur celles-ci (Cornell Empowerment 
"Les efforts qu'elles déploient pour restructurer la vie de certains survivants psychiatriques se heurtent au système de traitement psychiatrique dont les efforts quotidiens vont à l'encontre de ces objectifs d'émancipation..."
Group 1989: 1). L'entreprise génère des profits qui servent à rémunérer les employés et à la maintenir à flot.

Il ne suffit pas, toutefois, d'obtenir des contrats lucratifs. Un contrat avantageux pour l'entreprise peut être désastreux pour les employés. Le véritable succès d'une entreprise gérée par des survivants psychiatriques est fonction du nombre de personnes qu'elle emploie et du nombre d'heures de travail qu'elle fournit à chacun d'eux. Il est fonction de la durée de la période pendant laquelle les gens peuvent conserver leur emploi, de leur participation au fonctionnement de l'entreprise et de la possibilité qui leur est offerte de choisir leur mode de participation. Le succès est fonction du sentiment d'appartenance des membres, de leur participation à la prise de décisions (OCAB 1995).

Même si les entreprises gérées par les survivants psychiatriques s'acquittent convenablement de toutes ces responsabilités, il y a des limites à ce qu'elles peuvent accomplir. Les efforts qu'elles déploient pour restructurer la vie de certains survivants psychiatriques se heurtent au système de traitement psychiatrique dont les efforts quotidiens vont à l'encontre de ces objectifs d'émancipation (Rose et Black 1985). La majorité des employés continuent d'avoir des contacts avec ce système, ce qui influe sur leur état aussi bien physique que psychologique. Les entreprises gérées par des survivants psychiatriques ne peuvent annuler les effets continus de ce système; elles font tout au plus un pas dans cette voie. Il y a également des limites à la place qu'elles peuvent occuper, car le contexte économique national et mondial a une influence déterminante sur la situation économique locale.

Cet article a été publié par le Caledon Institute of Social Policy. Il est également disponible en anglais auprès de l'Institut au (613) 729-3340. Reproduit avec permission de l'auteure.

\section{Bibliographie}

BURSTOW,B. et D.Weitz (1988). Shrink Resistant :The Struggle Against Psychiatry in Canada,Vancouver, New Star Books.

CHURCH, K. (1997). "Business (not Quite) as Usual: Psychiatric Survivors and Community Economic Development in Ontario», E. Shragge (éd) Community Economic Development : In Search of Empowerment ( $2^{\mathrm{e}}$ éd), Montréal, Black Rose Press. 
CHURCH, K. (1993). Breaking Down/Breaking Through : Multi-Voiced Narratives on Psychiatric Survivor Participation in Ontario's Community Mental Health System, thèse de doctorat inédite, University of Toronto.

CHURCH, K. (1991). Re/Membering Ourselves: A Resource Book on Psychiatric Survivor Leadership Facilitation, Toronto, Psychiatric Survivor Leadership Facilitation Program.

CHURCH, K. (1987). «A Place in the Local Economy: The Haldimand-Norfolk Work Group of Simcoe», Forum \#11, Toronto, Association canadienne pour la santé mentale (nationale).

CHURCH, K. et L. CREAL (1995a). Voices of Experience : Five Tales of Community Economic Development in Toronto, Toronto, Toronto Community Economic Development Network.

CHURCH, K. et L. CREAL (1995b). Working the Margins: Qualitative Dimensions of Community Economic Development in Toronto, Toronto, Toronto Community Economic Development Network.

CONSUMER/SURVIVOR DEVELOPMENT INITIATIVE (1992). Policy Discussion Paper, Toronto, Consumer/Survivor Development Initiative.

CORNELL EMPOWERMENT GROUP. (1989). The Empowerment Process, Dissertation Van Leer, Ithaca, NY, Cornell University.

DAIN, N. (1989). "Critics and Dissenters: Reflections on 'Anti-Psychiatry' in the United States". Journal of the History of the Behavioral Sciences, 25 (janvier), 3-24.

HUTCHISON, P., J. LORD et L. OSBORNE-WAY. (1986). Participating With People Who Have Directly Experienced the Mental Health System, Toronto, Association canadienne pour la santé mentale (nationale).

ONTARIO (1988). Comité provincial de services communautaires de santé mentale. Renforcer le soutien communautaire à la population: Planification de la santé mentale en Ontario, Toronto, ministère de la Santé.

ONTARIO COUNCIL OF ALTERNATIVE BUSINESS (1995). Yes We Can! Promote Economic Opportunity and Choice Through Community Business, Toronto.

ONTARIO COUNCIL OF ALTERNATIVE BUSINESS (1994). Group Hallucinations : Overcoming Disbelief:Yes, You Can Start a Community Business!, Toronto.

O'REGAN, F. et J. CONWAY (1993). From the Bottom Up:Toward a Strategy for Income and Employment Generation Among the Disadvantaged, Washington, DC, The Aspen Institute.

PAPE, B. (1988). Consumer Participation: From Concept to Reality, Toronto, Association canadienne pour la santé mentale (nationale).

ROSE, S. et B. BLACK (1985). Advocacy and Empowerment: Mental Health Care in the Community, Boston, Routledge and Kegan Paul.

TRAINOR, J. et al. (1992). «Élaboration d'un modèle sectoriel d'une politique pour les personnes souffrant de maladie mentale grave», La santé mentale au Canada, vol. 40, no 1, 28-38.

TRAINOR, J. et K. CHURCH (1984). A Framework for Support, Toronto, Association canadienne pour la santé mentale (nationale).

WALLACE, B. (1996). "CED on the Street: Planning a Community Venture Comes Hard to an Association of Street People in Victoria», Making Waves, vol. 7, no 1, 16-18.

WILDMAN, P. (1996). «Action Learning and Community Economic Development», T. Carr (ed) Creative Applications of Action Learning and Research, Brisbane, ALARPM.

WILDMAN, P. (1993). Growing Your Own Community Economy, Queensland, Australia, Prosperity Press. 
Cet article est aussi disponible en anglais. La traduction, la publication et la distribution de cet article ont été rendus possible grâce à l'appui du Programme des partenariats communautaires de Patrimoine canadien.

\section{Notes}

1. Le présent document s'inspire d'un texte plus élaboré intitulé Because of Where We've Been :The Business Behind the Business of Psychiatric Survivor Economic Development, produit par l'Ontario Council of Alternative Businesses et la 761 Community Development Corporation. Les personnes intéressées peuvent se procurer ce document ainsi que plusieurs publications figurant dans la liste de références en s'adressant à l'un ou l'autre des deux organismes précités, à l'adresse suivante : 642 rue King Ouest, Toronto M5V 1M7. Tél. : (416) 406-3159 ou: (416) 703-5459.

2. Je tiens à souligner la contribution importante, aussi bien au contenu qu'au cheminement de mon travail, des survivants psychiatriques qui oeuvrent dans le domaine du développement communautaire. Je veux tout particulièrement remercier la Voices Exchange Team: Diana Capponi, coordonnatrice de l'Ontario Council of Alternative Businesses; Rick Ciccarelli, directeur administratif de la 761 Community Development Corporation; Laurie Hall, directrice administrative d'A-Way Express; et Mary Taylor, directrice administrative d'ABEL Enterprises. 